\title{
Thyroid cancer in childhood: Management strategy, including dosimetry and long-term results
}

\author{
Markus Luster, ${ }^{1}$ Michael Lassmann, ${ }^{1}$ Lutz S. Freudenberg, ${ }^{2}$ Christoph Reiners ${ }^{1}$ \\ ${ }^{1}$ Department of Nuclear Medicine, University of Würzburg, ${ }^{2}$ Department of Nuclear Medicine, University of Duisburg- \\ Essen, Germany
}

\begin{abstract}
Thyroid cancer, although very rare in childhood, represents the most common pediatric endocrine neoplasia. The low incidence and the resulting limited availability of prospective, randomized trials lead to a lack of evidence- based recommendations for treatment strategies. Total thyroidectomy and lymphadenectomy, when indicated, followed by ablative radioiodine treatment, are considered the cornerstones of initial patient management which decrease the risk of relapse. On the other hand, less aggressive treatment modalities should also be aimed at due to the high life expectancy of this special patient group and the potential impairment of the quality of life. These considerations have led to individualized "tailored" therapeutic approaches based on prior risk stratification. This article mainly deals with novel nuclear medicine concepts for dosing regimens in radioiodine therapy.
\end{abstract}

Key words: ${ }^{131}$ I dosimetry, Paediatrics, Positron emission tomography, Thyroid cancer

\section{INTRODUCTION}

Thyroid cancer accounts for the most frequent tumors of endocrine glands in children and adolescents; generally, however, thyroid cancer in this population is rare (incidence rate approximately 0.2 - 0.5 per 100,000 per year). The US Cancer Registry SEER (Surveillance Epidemiology and End Results) reports an annual age-adjusted incidence rate of 8.5 per 100,000 men and women, approximately $2.1 \%$ diagnosed under age 20 . These rates are based on cases

\footnotetext{
Address for correspondence:

Markus Luster, MD, Department of Nuclear Medicine, University of Würzburg, Josef-Schneider-Strasse 2, 97080 Würzburg GERMANY, Tel.: +49-931-201-35874, Fax: +49-931-201-35247,

e-mail: luster@nuklearmedizin.uni-wuerzburg.de Received 05-07-07, Revised 20-09-07, Accepted 01-10-07
}

diagnosed in 2000-2004. Analysis of corresponding data from a regional Tumor Registry in Würzburg, Germany are shown in Table 1.

\section{Treatment strategies and results}

The actual knowledge related to clinical courses and treatment strategies for childhood thyroid cancer have recently been summarized by Thompson and Hay. ${ }^{1}$ The most relevant data of these publications are shown in Tables $2 \mathrm{a}$ and $2 \mathrm{~b}$ (presenting the results of studies with more than 50 cases of childhood thyroid cancer each). First, it can be stated that thyroid cancer in children presents in more than $90 \%$ of the cases as the most favorable papillary type in terms of prognosis. A typical finding in pediatric cancers is tumor growth beyond the thyroid capsule, which may at least in part be attributed to the overall small 
Table 1. Data of an annual, age-adjusted incidence rate per 100,000 inhabitans from a regional Tumor registry in Würzburg, Germany $(\mathrm{n}=1188)$.

\begin{tabular}{llc}
\hline Adults & 6.9 & $(97.3 \%)$ \\
Children (0-9 yrs) & 0.02 & $(0.25 \%)$ \\
Children (10-14 yrs) & 0.04 & $(0.5 \%)$ \\
Adolescents $(15-19$ yrs) & 0.14 & $(2.0 \%)$ \\
Total (age <20 yrs ) & 0.20 & $(2.75 \%)$ \\
\hline
\end{tabular}

volume of the thyroid gland; therefore, these tumors will be classified as stage pT4 (approx. 30\% of the cases) according to the UICC TNM classification of 2002. Also characteristic of the disease is frequent regional lymph node involvement (approx. 70\% of the cases), while distant metastases - mainly to the lungs - are seen in approximately $20 \%$. Because of its often invasive growth, frequent lymph node metastases and relatively common distant spread, the initial presentation and the course of thyroid cancer in children is generally considered rather aggressive.

Thus, comparatively radical therapeutic concepts are recommended for treatment of childhood thyroid cancer $^{2-10}$ (Table 2). About three quarters of the experts are in favor of total or near-total operative resection of the thyroid, removal of neck lymph nodes and subsequent ablative radioiodine treatment for removal of potentially remaining thyroid and/or tumor tissue. Table $2 \mathrm{~b}$ shows that this radical surgical and radiotherapeutical approach has been followed, especially in recently published studies. Total removal of the thyroid by surgery and radioiodine treatment is followed by TSH-suppressive hormone replacement with levothyroxine.

The main risks of surgical treatment are related to potential laryngeal nerve palsy and hypoparathyroidism. Disturbances of vocal function - from hoarseness to aphonia-occur in up to $10 \%$ of the affected children. Recent data report up to $12 \%$ of children with thyroid cancer developing postoperative hypocalcemia because of impairment of the parathyroid glands. Parathyroid hormone deficiency and consequent hypocalcemia may result in paresthesia and muscle spasms. ${ }^{11,12}$

Radioiodine treatment is usually performed under endogenous TSH stimulation after thyroid hormone withdrawal. T4 clearance rates and serum TSH to FT4 ratio tends to be generally higher in children than in adults allowing for a shorter hypothyroid phase. Kuijt and Huang reported that TSH values $>25 \mathrm{mU} / \mathrm{l}$ are achieved within 14 days of levothyroxine withdrawal. ${ }^{13}$ Alternatively, exogenous TSH elevation may be accomplished by the administration of recombinant

Table 2a. Literature data (as summarized by Thomson and Hay (1)) includes studies with more than 50 cases each without and after radiation exposure (Chernobyl).

\begin{tabular}{|c|c|c|c|c|c|c|c|}
\hline Author & Year & Number of patients & Follow-up (Months) & PTC (\%) & pT4 $(\%)$ & N1 $(\%)$ & M1 (\%) \\
\hline \multicolumn{8}{|c|}{ No irradiation exposure } \\
\hline Schlumberger & 1987 & 72 & n.r. & n.r. & 67 & 90 & 18 \\
\hline Zimmermann & 1988 & 58 & $331 \mathrm{AM}$ & 100 & 24 & 90 & 7 \\
\hline LaQuaglia & 1988 & 103 & $240 \mathrm{Med}$ & 84 & 24 & 71 & 19 \\
\hline Vassilopoulou & 1998 & 112 & 112 Med & 92 & 38 & 63 & 28 \\
\hline Dottorini & 1997 & 85 & $111 \mathrm{Med}$ & 85 & 31 & 60 & 19 \\
\hline Segald & 1998 & 61 & n.r. & 79 & 26 & 49 & 6 \\
\hline Newman & 1998 & 329 & $136 \mathrm{Med}$ & 90 & 32 & 74 & 25 \\
\hline LaQuaglia & 2000 & 83 & $131 \mathrm{Med}$ & 90 & 48 & 90 & $100^{*}$ \\
\hline Jarzab & 2000 & 109 & $60 \mathrm{AM}$ & 71 & n.r. & 59 & 16 \\
\hline Grigsby & 2001 & 56 & $132 \mathrm{Med}$ & 95 & 54 & 60 & 13 \\
\hline \multicolumn{8}{|c|}{ Irradiation exposure } \\
\hline Rybakov & 2000 & 330 & $180 \mathrm{AM}$ & 94 & 55 & 62 & 15 \\
\hline Demidchik & 2006 & 741 & $97 \mathrm{AM}$ & 95 & 16 & 69 & 18 \\
\hline
\end{tabular}

PTC: papillary histology, pT4: invasive growth, N1: lymph node metastases, M1: distant metastases, AM: arithmetic mean, Med: median, n.r.: not reported 
Table 2b. Treatment and treatment related side effects in children and adolescents with thyroid cancer without and after radiation exposure by Chernobyl (literature data as summarized by Thomson and Hay ${ }^{1}$ ).

\begin{tabular}{|c|c|c|c|c|c|c|c|c|c|}
\hline Author & Year & $\begin{array}{c}\text { Thyroidectomy } \\
(\%)\end{array}$ & $\begin{array}{l}\text { Lymphadenectomy } \\
(\%)\end{array}$ & ${ }^{131} \mathbf{I}(\%)$ & LT4 (\%) & $\begin{array}{c}\text { Laryngeal } \\
\text { nerve palsy } \\
(\%) \\
\end{array}$ & $\begin{array}{c}\text { Calcium } \\
\text { deficiency } \\
(\%)\end{array}$ & $\begin{array}{l}\text { Mortality } \\
(\%)\end{array}$ & $\begin{array}{c}\text { Reccur- } \\
\text { rence } \\
(\%)\end{array}$ \\
\hline \multicolumn{10}{|c|}{ No irradiation exposure } \\
\hline Schlumberger & 1987 & 40 & n.r. & 22 & n.r. & 11 & 7 & n.r. & 58 \\
\hline Zimmermann & 1988 & 76 & 60 & 20 & mostly & 0 & 24 & 15 & 48 \\
\hline LaQuaglia & 1988 & 45 & 73 & 21 & 80 & 30 & 32 & 0 & 34 \\
\hline Vassilopoulou & 1998 & 72 & 62 & mostly & mostly & n.r. & 13 & 11 & 40 \\
\hline Dottorini & 1997 & 56 & n.r. & 89 & mostly & 4 & 8 & 0 & 7 \\
\hline Segald & 1998 & 83 & 59 & 100 & 100 & 10 & 10 & 3 & 33 \\
\hline Newman & 1998 & 54 & 56 & 43 & n.r. & 2 & 12 & 1 & 33 \\
\hline LaQuaglia & 2000 & 66 & 51 & 100 & 100 & 1 & 12 & 0 & 31 \\
\hline Jarzab & 2000 & 100 & 100 & 100 & 100 & 10 & 6 & 0 & 15 \\
\hline Grigsby & 2001 & 92 & 95 & 82 & 100 & 0 & 7 & 0 & 34 \\
\hline \multicolumn{10}{|c|}{ Irradiation exposure } \\
\hline Rybakov & 2000 & 84 & 57 & 75 & 100 & 12 & 6 & 2 & 7 \\
\hline Demidchik & 2006 & 58 & 82 & 63 & 100 & 6 & 12 & 1 & 28 \\
\hline
\end{tabular}

n.r.: not reported

human Thyrotropin.

However, the vast preponderance of clinical investigation and all regulatory approval for rhTSH have been in adult patients (18 years and older). Only a few reports have been published on rhTSH use in children and adolescents, most of which are case reports ${ }^{14}$ or relatively small single-center ${ }^{15,16}$ or two-center ${ }^{17}$ series.

Radioiodine therapy is administered as a capsule in children, special attention needing to be paid to adjuvant antiemetic medication.

The dosing regimen that was developed in our institution and successfully followed in more than 200 patients based on individual body weight is shown in Table 3.

Table 3. Dosing regimen based on individual's body weight.

\begin{tabular}{lccc}
\hline & $\begin{array}{c}\text { Activity per } \\
\text { Therapy }\end{array}$ & $\begin{array}{c}\text { Time } \\
\text { Interval } \\
\text { between } \\
\text { Therapies }\end{array}$ & $\begin{array}{c}\text { Mean } \\
\text { Total } \\
\text { Activity }\end{array}$ \\
\hline Ablation (M0) & $50 \mathrm{MBq} / \mathrm{kg} \mathrm{BW}$ & $6,3 \pm 2,4$ mos. $5,8 \pm 4,1 \mathrm{GBq}$ \\
Therapy (M1) & $100 \mathrm{MBq} / \mathrm{kg} \mathrm{BW}$ & $5,7 \pm 2,1$ mos. & $\begin{array}{c}13,4 \pm 6,2 \\
\text { GBq }\end{array}$ \\
\hline
\end{tabular}

${ }^{131}$ I treatment may lead to temporary decreases of salivary flow and disturbances of taste (in up to $30 \%$ of the cases). ${ }^{12}$ Permanent xerostomia, however, is less frequent. Conversely, mild to moderate nausea during the first days after radioiodine administration is quite frequent in children. The most severe longterm side effect of radioiodine treatment is considered to be radiation induced leukemia which occurs in approximately $1 \%$ of the patients. In children and adolescents with diffuse pulmonary metastases and intensive radioiodine uptake, pulmonary fibrosis may develop in $1 \%$ of the cases. ${ }^{12}$

In male adolescents and young adults radioiodine can impair the quality of sperm thus compromising fertility. Mainly in cases of repeated high-dose therapy sperm banking should be considered. ${ }^{18}$

In spite of the apparently aggressive course of thyroid cancer in children, treatment results are favorable (Table 2b). Cancer-related mortality in children and adolescents is in the range of $1 \%$; however, relatively frequent tumor recurrences, which may occur after years or even decades, are encountered on long-term follow-up. Thus lifelong, intensive aftercare in an experienced center is highly recommended, since in the case of relapse, repeated surgery and/or radioiodine 
treatment may be initiated for secondary induction of complete remission.

Recently, the results of treatment of thyroid cancer in children and adolescents have been investigated in a large retrospective study by Handkiewicz-Junak et al. ${ }^{19}$ The authors were able to demonstrate that near-total or total thyroidectomy and adequate lymphadenectomy for lymph node metastasis significantly and independently decrease the risk of locoregional recurrence. Multivariate analysis of this group of children after an extended observation time also revealed a statistically significant benefit from postoperative radioiodine therapy.

Corresponding findings of children who developed the disease after the reactor catastrophe of Chernobyl have been published by two groups from Kiev, Ukraine ${ }^{20-22}$ and Minsk, Belarus. ${ }^{23}$

Table 2a-2b compares the data of those two Centers, which include a large number of thyroid cancer cases in children and adolescents (330 and 741 patients, respectively) with the data of spontaneous (no known irradiation exposure) cases published in the literature. The therapeutic strategies which have been followed by the two groups are in good agreement with the recommendations in the recent literature. However, radioiodine treatment has been used less frequently due to the lack of availability of this treatment modality in those countries.

Figure 1 shows the whole-body scan of a 15-year old girl from Belarus with locally invasive thyroid cancer and metastases to the lymph nodes and to the lungs. Immediately after the first course of radioiodine therapy, the tumor, and the lymph node and lung metastases are clearly visible on the scan. Follow-up diagnostic scintigraphy performed two years later proves the efficacy of radioiodine therapy: the lesion in the thyroid bed, and regional and distant metastases have been successfully eradicated (the accumulation of radioiodine in the gastrointestinal tract and the urinary bladder are physiological).

A detailed analysis of the very large patient cohort from Minsk (741 cases) shows that 5- and 10-year survival rates are close to $100 \%(99.3 \%$ and $98.5 \%$, respectively). ${ }^{23}$ In a subgroup consisting of 104 children and adolescents who have been treated with radioiodine because of pulmonary metastases at our tertiary referral center in Würzburg, Germany, complete remissions have been observed in $28 \%$ as defined by negative imaging and the tumor marker thyroglobulin below detection limit, stable partial remission in $37 \%$ (whole-body imaging negative, tumor marker thyroglobulin between 1 and $10 \mathrm{ng} / \mathrm{ml}$ ) and partial remissions in $35 \%$ (positive whole-body scan and/or thyroglobulin above $10 \mathrm{ng} / \mathrm{ml}$ ). Importantly, not a single case displayed disease progression.

\section{RECENT STRATEGIES FOR TREATMENT OF THYROID CANCER IN CHILDREN AND ADOLESCENTS:}

\section{Dosimetry Concepts}

The goal of individualized radioactive iodine

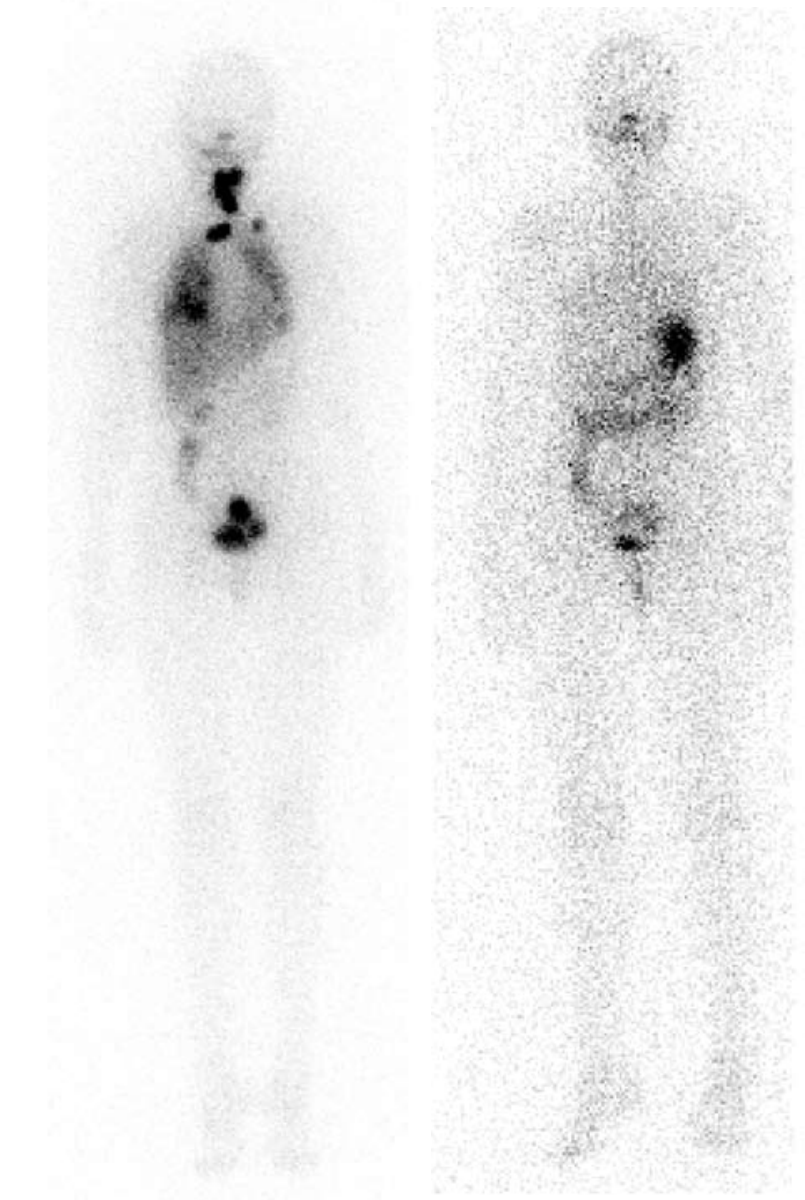

Figure 1. Whole-body scan of a 15 -year old girl with metastatic DTC before (left) and after (right) effective radioiodine therapy. 
therapy (RIT) is to deliver ample radiation doses to destroy thyroid remnants, tumor foci, or both, without exceeding safe doses to the whole body, particularly the bone marrow and lungs. Consequently, pretherapeutic dosimetry allows individual assessment of radiation exposure with the rationale to optimize the risk/benefit ratio of RIT. This approach is of special interest in pediatric DTC patients who tend to have highly radiosensitive, bulky and widespread tumor ${ }^{9,15,16}$ and a heightened risk of long-term radiation-related sequelae.

Presently there are three concepts as to how to determine which activity should be administered for the radioiodine therapy of DTC: $:^{24}$

\section{a) Fixed Activities}

There is a broad range of fixed activities of ${ }^{131} \mathrm{I}$ recommended to be administered in adults (see e.g. 25,26 ). Usually an activity between 1.1 and $3.7 \mathrm{GBq}$ is prescribed in adults for the first radioiodine therapy after thyroidectomy in newly diagnosed DTC to ablate the remaining glandular tissue. Higher amounts of ${ }^{131} \mathrm{I}$ are given in subsequent therapies or in the case of metastatic disease. Usually the activity is limited for safety to around 7.4 GBq. For a survey on the use of fixed activities for the treatment of DTC, see the review by van Nostrand et al. ${ }^{24}$

The protocol for children from Belarus treated in our institution included the administration of 50 $\mathrm{MBq} / \mathrm{kg}$ bodyweight for remnant ablation and 100 $\mathrm{MBq} / \mathrm{kg}$ for treatment of metastases.

The main disadvantage in using a fixed activity approach is the failure to consider the individuality of the patient. The "ideal" activity of radioiodine to treat metastatic thyroid carcinoma is the lowest possible amount of radioiodine that delivers a lethal dose of radiation to the entire lesion/metastasis while minimizing side effects. Empiric fixed activities by their very nature make no attempt to determine either the minimal radioiodine that will deliver a lethal dose or the maximum allowable reasonably safe absorbed dose.

\section{b) Lesion-based Dosimetry}

The objective of remnant or lesion dosimetry is to determine the radioiodine activity that delivers the intended absorbed dose of radiation to ablate thyroid remnant or to treat metastatic disease while minimizing the risk to the patient; the doses are traditionally considered to be 300 and $80 \mathrm{~Gy}$, respectively. ${ }^{27,28}$ In contrast to these pre-therapeutic dosimetric procedures, the radiation absorbed dose delivered by the ${ }^{131}$ I therapeutic activity itself to the thyroid remnant or to other lesions also may be measured post-therapy, attempting to correlate this dose with clinical results.

In order to perform these calculations, it is necessary to measure pre-therapeutically the uptake and clearance of the ${ }^{131} \mathrm{I}$ from identifiable thyroid remnants and/or metastatic lesions.

To determine the concentration of ${ }^{131} \mathrm{I}$, one needs to know how much activity is contained in the lesion. One way in which this can be achieved is based on an analysis of selected regions of interest on conjugate view gamma camera images. ${ }^{29}$ These images are obtained at several time points measured from the time of administration of a tracer activity. Typically, these measurements are performed up to 96 hours, but later time samples might be necessary if the uptake and clearance are delayed. A curve-fitting procedure is then used to determine the assumed single-exponential half-life value and to extrapolate the curve to zero time in order to determine the initial activity in the lesion.

Specifically, when using probe data or planar scintigrams, another parameter needed for calculating the particular activity is the mass of the lesion. For ablation therapies, the various procedures for determining the remnant size such as ultrasound or CT are unreliable, as no validated method exists to exactly determine the mass of thyroid remnants after surgery. ${ }^{30}$ For this reason one has to be careful when reporting absorbed doses by the target tissue.

\section{c) Bone Marrow Limited Approach}

The method originally reported by Benua and Leeper ${ }^{31,32}$ allows an estimate of the radiation absorbed dose that will be delivered to the haematopoetic system from each GBq administered to any patient. This is possible because it involves the collection of data over the course of 4 or more days after the administration of a tracer activity of ${ }^{131} \mathrm{I}$ to the patient. 
The measurement procedures involve serial blood sampling and serial measurements of the patient's whole body activity using a calibrated probe.

In the classic Benua approach, the blood is considered the critical organ that is irradiated either from the particles emitted from activity in the blood itself or from the emissions originating from activity dispersed throughout the remainder of the body.

Details of the sampling frequency of the data and of the calculation process can be found in the paper by van Nostrand et $\mathrm{al}^{24}$ for the classical Benua approach and in Hänscheid et al for a refined patient specific method. ${ }^{30}$ Data should be collected for more than $100 \mathrm{hrs}$ after administration.

Recently, in the framework of several international multicenter trials for the study of the biokinetics after the use of recombinant human $\mathrm{TSH},{ }^{30,33}$ the absorbed dose to the blood was calculated with a modified method deduced from a procedure originally described by Thomas et al..$^{34}$

An example of a pre-therapeutic dose calculation can be found in Figures 2 and 3. Figure 2 shows the

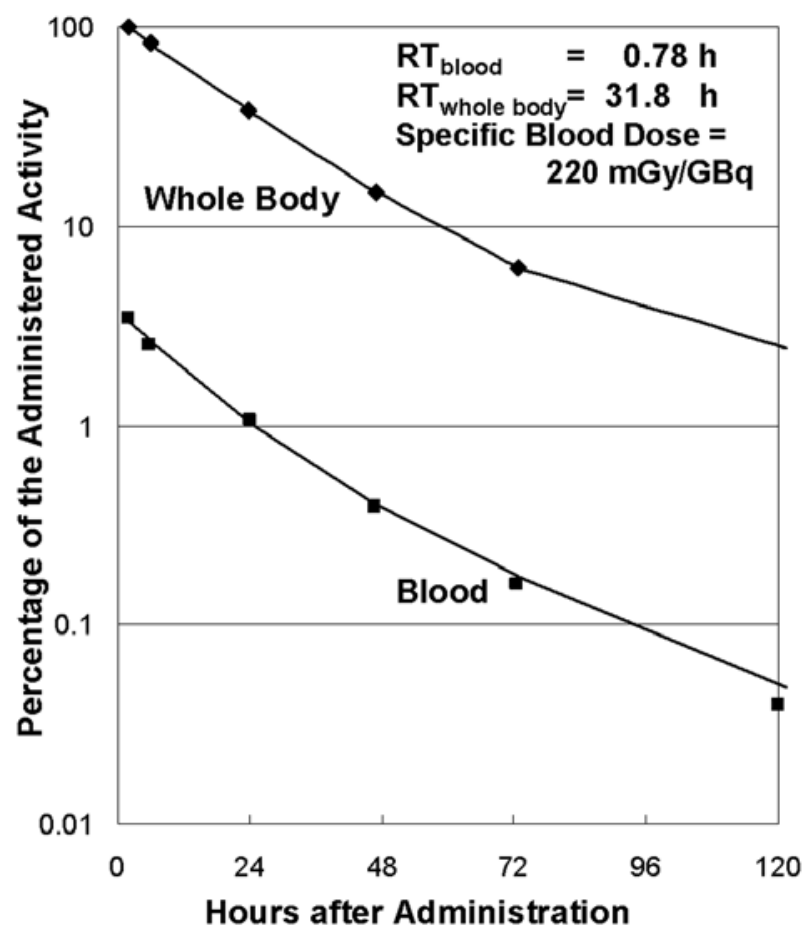

Figure 2. Example of a pre-therapeutic whole-body and blood dose calculation. iodine biokinetics of $5 \mathrm{MBq}{ }^{131}$ I for blood and whole body. The corresponding residence times and specific blood doses are shown in Figure 3. The two scans on the left side of Figure 3 show the $6 \mathrm{hr}$ and $72 \mathrm{hr}$ measurement of the neck.

Nine GBq were administered to this patient; the resulting post-therapy scan is shown on the right side of Figure 3.

The strengths of the blood-based approach are:

- determination of the maximal allowable safe activity of radioiodine in each patient individually, thereby avoiding a possible undertreatment,

- identification of patients whose maximal tolerable safe dose of radioiodine is less than the empiric fixed activities,

- the potential to give higher radiation doses to metastases at one time instead of multiple lower empiric doses with potentially lower effective radiation doses,

- a long history of use in several institutions.

The limitations of the blood-based approach are:

- increased cost and inconvenience,

- the absorbed dose to the metastases is not estimated and thus one may be giving the maximal allowable safe dose but not gaining any relevant therapeutic effect,

- to date, no systematic clinical data in children are available,

- present dosimetric approaches use ${ }^{131} I$ diagnostically, which has the potential to reduce the uptake of the therapeutic activity and thus reduce the radiation absorbed dose delivered to the metastasis. Pre-therapeutic ${ }^{131} \mathrm{I}$ activities of more than $10 \mathrm{MBq}$ should not be administered in order to avoid complication of the therapy by "stunning" introduced by the tracer activity mentioned earlier, ${ }^{35}$

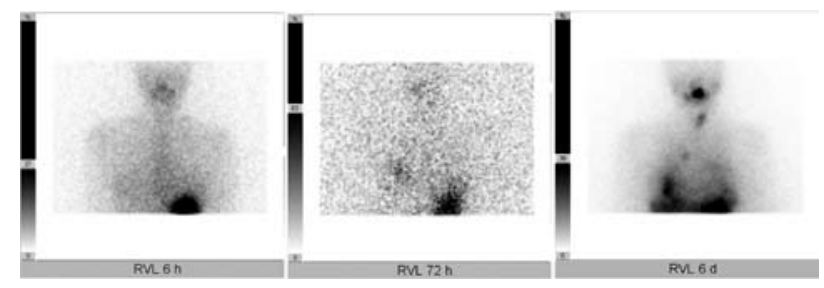

Figure 3. Example of $6 \mathrm{hr}$ and $72 \mathrm{hr}$ measurements of the neck and post-therapy scan. 
- there are no valid clinical data on the applicability of this method to lesions/metastases with high uptake $(\sim 5 \%)$,

- simplifications to the protocol have not yet been tested.

\section{Future perspectives}

\section{${ }^{124}$ I Positron Emission Tomography}

${ }^{124}$ I positron emission tomography/computed tomography dosimetry is increasingly used to calculate radioiodine therapy activities in patients with differentiated thyroid cancer. ${ }^{36-39}$ Several studies have shown the advantages of ${ }^{124} \mathrm{I}$ PET/CT dosimetry in adult DTC patients; ${ }^{36-39}$ however, until now no published study reports results of ${ }^{124} \mathrm{I}$ PET/CT dosimetry in pediatric patients.

Today's state of the art of PET ${ }^{124}$ I based thyroid dosimetry has been described in a recent publication by Sgouros et $\mathrm{al}^{38}$ in which the authors showed, by using the PET results as input to a fully 3D dose planning program, spatial distributions of absorbed doses, isodose contours, dose-volume histograms and mean absorbed dose estimates for a total of 56 tumors in 15 patients. The mean absorbed dose values for individual tumors ranged from 1.2 Gy to $540 \mathrm{~Gy}$. The absorbed dose distribution within individual tumors was widely distributed, ranging from a minimum of 0.3 Gy to a maximum of 4,000 Gy, showing the high variability of dose ranges even in the same patient.

${ }^{124} \mathrm{I} \mathrm{PET} / \mathrm{CT}$ dosimetry procedure requires serial PET scans, gamma-camera scans and blood-sampling up to five consecutive days. ${ }^{39,40}$ Whole-body data are acquired at 4, 24, 48, 72 and $96 \mathrm{hr}$, and ${ }^{124} \mathrm{I} \mathrm{PET/CT}$ data $25 \mathrm{hr}$ post administration of $22-26 \mathrm{MBq}{ }^{124} \mathrm{I}^{41}$ under TSH stimulation. Whole-body emission data are obtained by scanning from head to thigh. With the ${ }^{124}$ I PET data, each patient's remnant or lesion dose(s) per GBq of administered ${ }^{131} \mathrm{I}$ can be determined.

Furthermore, each patient's maximum activity of ${ }^{131}$ I avoiding a $>2$ Gy dose to the blood is calculated. To do so, activity-time curves of the blood and wholebody kinetics are used. ${ }^{124} \mathrm{I}$ PET/CT findings may serve as key but not exclusive inputs into the choice of RIT activity and other interventions. ${ }^{39}$

In a pilot study, 4 pediatric patients from the University-Hospital of Essen, where the same TSH stimulation and dosimetric methods, including the ${ }^{124} \mathbf{I}$ activity generally employed in adult patients, ${ }^{39}$ were analyzed. An example of a patient where ${ }^{124} \mathrm{I}$ PET/CT dosimetry allowed individualizing RIT by employing an activity to efficiently treat the metastases without exceeding the 2 Gy limit is demonstrated in Figure 4. It shows a patient with multiple iodine-avid metastases who received $10 \mathrm{GBq}{ }^{131} \mathrm{I}$ for first RIT due to the dosimetry results. Follow-up examinations 4 months post RIT showed successful treatment without iodine-uptake in follow-up ${ }^{124} \mathrm{I}$ PET and a decrease of Thyroglobulin from $430 \mathrm{ng} / \mathrm{ml}$ to $1.4 \mathrm{ng} / \mathrm{ml}$.

${ }^{124} \mathrm{I}$ PET/CT dosimetry is not only a promising tool in therapy planning in DTC patients; it has furthermore the potential to modify the management or better delineate the extent of disease, e.g. in the case of lymph node metastasis potentially treatable with surgery instead of RIT (see Figure 5 as example). However, further studies are required to appraise the clinical usefulness and impact on patient outcome, especially in pediatric DTC patients.

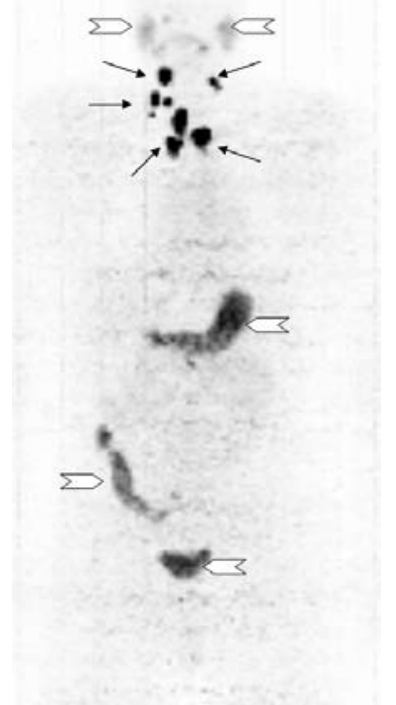

Figure 4. ${ }^{124} \mathrm{I}$-PET image (coronar view, $24 \mathrm{~h}$ post oral administration of $25 \mathrm{MBq}{ }^{124} \mathrm{I}$ ) to a 16-year old male patient with papillary DTC showing multiple iodine-avid cervical and mediastinal metastases (arrows). Note physiological uptake/accumulation of ${ }^{124} \mathrm{I}$ in salivary glands, stomach, colon and bladder (triangles). 


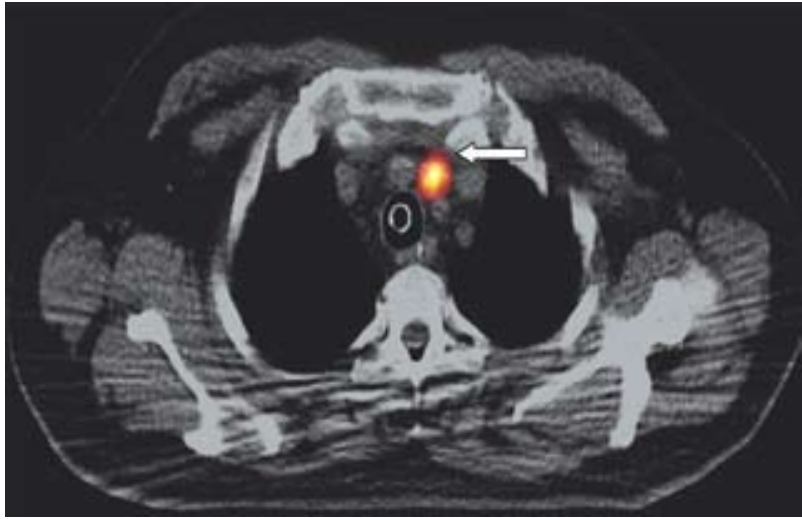

Figure 5. ${ }^{124} \mathrm{I}-\mathrm{PET} / \mathrm{CT}$ images of a 14 -year old female patient with an iodine-positive left mediastinal lymph node metastasis $0.9 \mathrm{~cm}$ in its largest diameter barely visible in CT alone (arrow). As curative radioiodine therapy was impossible due to insufficient iodine uptake, ${ }^{124} \mathrm{I}-\mathrm{PET} / \mathrm{CT}$ was used to plan surgery. Histology confirmed DTC metastasis.

\section{CONCLUSIONS}

Thyroid cancer is more aggressive in children and adolescents with regard to clinical course. Clear differences between the basic clinical features of radiation induced and spontaneous cases have not been observed. Using established treatment regimes - consisting of surgery, radioiodine therapy and thyroid hormone replacement - even advanced stages of childhood thyroid cancer can be treated effectively. Mortality of thyroid cancer in children and adolescents is low (1-2\%); this holds true even for radiation induced cases. However, it must be considered that recurrences of thyroid cancer can develop after years or even decades. For this reason - and for treatment of therapy related complications - lifelong, strict follow-up is mandatory. This particular group of patients should be followed up in specialized centers.

With regard to therapy, in the majority of patients the fixed activity based approach for the treatment of thyroid cancer may have its benefits due to its simplicity. In most cases this therapeutic regimen stays well below any toxicity limits. The main disadvantage in using this approach is the failure to consider the individuality of the patient. It also might over, or more probably under treat an individual patient without this being realized. In addition, the efficacy of several treatments in one patient might be degraded.

In the case of lesion-based dosimetry, the pre- therapeutic use of ${ }^{124}$ I seems to be the state of the art, although the matter of stunning has not been settled. In addition, careful PET scanner calibration is mandatory. In the lesion based approach the pre-therapeutic or therapeutic administration of radioactive iodine does not take into account regional differences in the local iodine uptake.

Patient-specific blood-based dosimetry is comparatively easy to perform before and during therapy. In selected patients this procedure will allow extending the activity beyond the limit of therapies using fixed activities and will reduce the risk of severe side effects.

In applying any of the above-mentioned methods, "stunning" should be avoided. For dosimetry purposes the pre-therapeutic use of ${ }^{123}$ I cannot be generally recommended due to the short half-life of this isotope.

\section{REFERENCES}

1. Thompson GB, Hay ID, 2004 Current Strategies fór Surgical Management and Adjuvant Treatment of Childhood Papillary Thyroid Carcinoma. World J Surg 28: 1187-1198.

2. Schlumberger M, De Vathaire F, Travagli JP, et al, 1987 Differentiated thyroid carcinoma in childhood: long-term follow-up of 72 patients. J Clin Endocrinol Metabol 65: 1088-1094.

3. Zimmerman D, Hay ID, Gough IR, et al, 1988 Papillary thyroid carcinoma in children and adults: long-term follow-up of 1039 patients conservatively treated at one institution during three decades. Surgery 104: 1157-1166.

4. LaQuaglia MP, Corbally MT, Heller G, et al, 1988 Recurrence and morbidity in differentiated thyroid carcinoma in children. Surgery 104: 1149-1156.

5. Vassilopoulou-Sellin R, Goepfert H, Raney B, et al, 1998 Differentiated thyroid cancer in children and adolescents: clinical outcome and mortality after long-term follow-up. Head Neck 20: 549-555.

6. Segald K, Shvero J, Stern Y, et al, 1998 Surgery of thyroid cancer in children and adolescents. Head Neck 20: 293-297.

7. Newman KD, Black T, Heller G, et al, 1998 Differentiated thyroid cancer: determinants of disease progression in patients $<21$ years of age at diagnosis: a report from the Surgical Discipline Committee of the Children's Cancer Group. Ann Surg 227: 533-541.

8. LaQuaglia MP, Black T, Holcomb GW, et al, 2000 Differentiated thyroid cancer: clinical characteristics, treatment and outcome in patients under 21 years of age 
who present with distant metastases: a report from the Surgical Discipline Committee of the Children's Cancer Group. J Pediatr Surg 35: 955-960.

9. Jarzab B, Junak DH, Wloch J, et al, 2000 Multivariate analysis of prognostic factors for differentiated thyroid carcinoma in children. Eur J Nucl Med 27: 833-841.

10. Grigsby PW, Galor A, Michalski JM, et al, 2002 Childhood and adolescent thyroid carcinoma. Cancer 95: 724-729.

11. Bohrer T, Pasteur I, Lyutkevych O, Fleischmann P, Tronko M, 2005 Permanenter Hypoparathyreoidismus infolge von Schilddrüsenkarzinomoperationen nach Tschernobyl in der Ukraine. Dtsch Med Wochenschr 130: 2501-2506.

12. Van Santen HM, Aronson DC, Vulsma T, et al, 2004 Frequent adverse events after treatment for childhoodonset differentiated thyroid carcinoma: a single institute experience. Eur J Cancer 40: 1743-1751.

13. Kuijt WJ, Huang SA, 2005 Children with differentiated thyroid cancer achieve adequate hyperthyrotropinemia within 14 days of levothyroxine withdrawal. J Clin Endocrinol Metab 90: 6123-6125.

14. Ralli M, Cohan P, Lee K, 2005 Successful use of recombinant human thyrotropin in the therapy of pediatric well-differentiated thyroid cancer. J Endocrinol Invest 28: 270-273.

15. Hoe FM, Charron M, Moshang T Jr, 2006 Use of the recombinant human TSH stimulated thyroglobulin level and diagnostic whole body scan in children with differentiated thyroid carcinoma. J Pediatr Endocrinol Metab 19: 25-30.

16. Lau WF, Zacharin MR, Waters K, Wheeler G, Johnston V, Hicks RJ, 2006 Management of paediatric thyroid carcinoma: recent experience with recombinant human thyroid stimulating hormone in preparation for radioiodine therapy. Intern Med J 36: 564-570.

17. Iorcansky S, Herzovich V, Qualey RR, 2005 Tuttle RM Serum thyrotropin (TSH) levels after recombinant human TSH injections in children and teenagers with papillary thyroid cancer. J Clin Endocrinol Metab 90: 6553-6555.

18. Krassas GE, Pontikides N, 2005 Gonadal effect of radiation from 131I in male patients with thyroid carcinoma. Arch Androl 51: 171-175.

19. Handkiewicz-Junak D, Wloch J, Roskosz J, et al, 2007 Total thyroidectomy and adjuvant radioiodine treatment independently decrease locoregional recurrence risk in childhood and adolescent differentiated thyroid cancer. J Nucl Med 48: 879-888.

20. Rybakov SJ, Komissarenko IV, Tronko ND, et al, 2000 Thyroid Cancer in Children of Ukraine after the Chernobyl Accident. World J Surg 24: 1446-1449.

21. Tronko MD, Bogdanova TI, Komissarenko IV, et al, 1999 Thyroid Carcinoma in Children and Adolescents in Ukraine after the Chernobyl Nuclear Accident. Cancer 86: 149-156.

22. Oliynyk V, Epshtein O, Sovenko T, et al, 2001 Postsurgical ablation of thyroid residues with radioiodine in Ukrainian children and adolescents affected by postChernobyl differentiated thyroid cancer. J Endocrinol Invest 24: 445-447.

23. Demidchik YE, Demidchik EP, Reiners Chr, et al, 2006 Comprehensive Clinical Assessment of 741 Operated Pediatric Thyroid Cancer Cases in Belarus. Ann Surg 243: 525-532.

24. Van Nostrand D, Atkins F, Yeganeh F, Acio E, Bursaw R, Wartofsky L, 2002 Dosimetrically determined doses of radioiodine for the treatment of metastatic thyroid carcinoma Thyroid 12: 121-134.

25. EANM, 2003 EANM procedure guidelines for therapy with iodine-131. Eur J Nucl Med Mol Imaging 30: BP2731.

26. Meier DA, Brill DR, Becker DV, et al, Society of Nuclear Medicine, 2002 Procedure guideline for therapy of thyroid disease with (131)iodine. J Nucl Med 43: 856-861.

27. Maxon HR, Thomas SR, Hertzberg VS, et al, 1983 Relation between effective radiation dose and outcome of radioiodine therapy for thyroid cancer. N Engl J Med 309: 937-941.

28. Maxon HR, Smith HS, 1990 Radioiodine-131 in the diagnosis and treatment of metastatic well differentiated thyroid cancer. Endocrinol Metab Clin North Am 19: 685-718.

29. Siegel JA, Thomas SR, Stubbs JB, et al, 1999 MIRD pamphlet no. 16: Techniques for quantitative radiopharmaceutical biodistribution data acquisition and analysis for use in human radiation dose estimates. J Nucl Med 40: $37 \mathrm{~S}-61 \mathrm{~S}$.

30. Hänscheid H, Lassmann M, Luster M, et al, 2006 Iodine biokinetics and dosimetry in radioiodine therapy of thyroid cancer: procedures and results of a prospective international controlled study of ablation after rhTSH or hormone withdrawal. J Nucl Med 47: 648-654.

31. Benua RS, Cicale NR, Sonenberg M, Rawson RW, 1962 Relation of radioiodine dosimetry to results and complications in treatment of metastatic thyroid cancer. American Journal of Roentgenology 87: 171-182.

32. Leeper RR, 1985 Thyroid cancer. Med Clin North Am 69: 1079-1096.

33. Luster M, Sherman SI, Skarulis MC, et al, 2003 Comparison of Radioiodine Biokinetics Following the Administration of Recombinant Human Thyroid Stimulating Hormone (rhTSH) and After Thyroid Hormone Withdrawal in Thyroid Carcinoma. Eur J Nucl Med Mol Imaging 30: 1371-1377.

34. Thomas SR, Samaratunga RC, Sperling M, Maxon HR 3rd, 1993 Predictive estimate of blood dose from external counting data preceding radioiodine therapy for thyroid cancer Nucl Med Biol 20: 157-162.

35. Lassmann M, Luster M, Hänscheid H, Reiners C, 2004 Impact of ${ }^{131} \mathrm{I}$ diagnostic activities on the biokinetics of thyroid remnants J Nucl Med 45: 619-625.

36. Pentlow KS, Graham MC, Lambrecht RM, et al, 1996 Quantitative imaging of iodine-124 with PET. J Nucl 
Med 37: 1557-1562.

37. Eschmann SM, Reischl G, Bilger K, et al, 2002 Evaluation of dosimetry of radioiodine therapy in benign and malignant thyroid disorders by means of iodine-124 and PET. Eur J Nucl Med Mol Imaging 29: 760-767.

38. Sgouros G, Kolbert KS, Sheikh A, et al, 2004 Patientspecific dosimetry for ${ }^{131} \mathrm{I}$ thyroid cancer therapy using 124I PET and 3-dimensional-internal dosimetry (3D-ID) software. J Nucl Med 45: 1366-1372.

39. Freudenberg L, Jentzen, W, Goerges R, et al, 2007 124I-
PET dosimetry in advanced differentiated thyroid cancer. Therapeutic impact. Nuklearmedizin 46: 121-128.

40. Freudenberg LS, Bockisch A, Jentzen W $2005{ }^{124}$ I Positron Emission Tomographic Dosimetry and Position Emission Tomography/Computed Tomography Imaging in Differentiated Thyroid Cancer. In: Biersack H, Grünwald F (eds) Thyroid Cancer, Springer; Berlin.

41. Knust EJ, Dutschka K, Weinreich R, 2000 Preparation of ${ }^{124} \mathrm{I}$ solutions after thermodistillation of irradiated 124TeO2 targets. Appl Radiat Isot 52: 181-184. 